EXTENDED REPORT

\title{
Doppler ultrasound findings in healthy wrists and finger joints
}

\author{
L Terslev, S Torp-Pedersen, E Qvistgaard, P von der Recke, H Bliddal
}

Ann Rheum Dis 2004;63:644-648. doi: 10.1136/ard.2003.009548

See end of article for authors' affiliations

.....................

Correspondence to: Dr L Terslev, The Parker Institute, Department of Rheumatology,

Frederiksberg Hospital, DK 2000 Frederiksberg, Denmark; lene.terslev@fh. hosp.dk

Accepted 6 August 2003
Objective: To evaluate the presence of flow by Doppler ultrasound (DUS) in the wrist and finger joints (carpometacarpal $1(\mathrm{CMC1})$, metacarpophalangeal (MCP), and proximal interphalangeal (PIP) joints) of healthy controls.

Methods: Twenty seven healthy volunteers (15 women, 12 men; mean age 45 years, range 18-93) with a total of 324 joints were examined by DUS. The synovial vascularisation was determined by colour Doppler and the spectral Doppler resistive index (RI). Patients were only included if no synovitis was suspected at the clinical examination and the values for biochemical analysis were within the normal range.

Results: We found colour pixels in 15 of the 27 examined wrist scans and in 8 of these a measurable RI. In the $\mathrm{CMCl}$ joint, colour pixels were found in 11 of the 27 scans and in 9 of these a measurable RI. For the MCP joints, colour pixels were found in 10 out of 135 scans and in 3 of these a measurable RI. The mean RI for all three types of joints was 0.85 and the mean pixel fraction varied from 0.05 to 0.08 . Only one PIP joint scan was found to have colour pixels and a flow with an RI of 0.67.

Conclusion: Synovial vascularisation may be detected in healthy subjects using DUS. Newer US machines have higher Doppler sensitivity, and it is necessary to be able to distinguish normal from pathological synovial flow.
D oppler ultrasound (DUS) is increasingly used in the evaluation of joint inflammation and has the advantage of giving an objective analysis of the disease without the use of ionising radiation. ${ }^{1}$ It is accessible in many outpatient clinics, has no contraindications, and poses no problems regarding patient compliance. Colour and power Doppler have been used to identify hyperaemia associated with inflammation ${ }^{2-5}$ by visualising the vascularity in the inflamed synovial membrane. DUS can detect synovitis in arthritis, and several studies in patients with rheumatoid arthritis (RA) have shown that it can detect treatment response following glucocorticosteroid administration ${ }^{6-8}$ and after systemic treatment with biologicals such as tumour necrosis factor- $\alpha .{ }^{9}{ }^{10}$ Because the newer US machines are equipped with high frequency transducers (10-20 MHz) and increased Doppler sensitivity, it has become possible to detect vascularisation even in a normal synovial membrane, as previously shown in the elbow joint. ${ }^{11}$ Both the detection limits and the reference range for DUS in normal synovial perfusion have yet to be established.

As the wrist and fingers are frequently targeted in RA, the aim of this study was to examine the wrist and finger joints of healthy volunteers by DUS for presence of colour pixels on the scan (indicating perfusion) and to evaluate the flow profile of vessels if detected, in the normal synovial membrane.

\section{MATERIALS AND METHODS \\ Subjects}

Staff members in our clinic and their families made up the 28 subjects we recruited to represent predefined age groups of 10 year intervals from 15-65 years or above. A total of 27 healthy volunteers ( 15 women, 12 men; mean age 45 years, range 18-93) were included in the study. The subjects were not currently engaged in heavy manual labour or performing sports activities with their hands, had no history of arthritis or hand or wrist trauma, and were not experiencing current symptoms. None of the subjects had clinical signs of inflammatory or degenerative joint diseases. A total of 324 joints was examined ( 12 joints per subject): the wrist, carpometacarpal l (CMCl), metacarpophalangeal (MCP) 15 , and proximal interphalangeal (PIP) $1-5$ joints.

The study was approved by the local ethics committee and written informed consent was obtained from all participants.

\section{Clinical examination}

The joints were assessed clinically with a score of each joint for the degree of tenderness and swelling from 0 to 3 . The subject filled in a Health Assessment Questionnaire (HAQ), indicated pain on a visual analogue scale (VAS) from 0$100 \mathrm{~mm}$, and reported morning stiffness in minutes. Biochemical measures were obtained the same day as erythrocyte sedimentation rate (ESR) and C-reactive protein (CRP). The mean ESR was 7 (range 1-18) and the mean CRP was $2.0(0.4-7.7)$.

The volunteers were only included if no synovitis was suspected clinically, and the values for ESR and CRP were within the normal range. One subject was not included because of elevated ESR and CRP, probably caused by a recent knee operation.

\section{Ultrasound}

An Acuson Sequoia ${ }^{\circledR}$ US system (Mountainview, CA, USA) was used for the US examinations using a $15 \mathrm{MHz}$ linear array transducer. The healthy volunteers were examined in upright position with the hand of interest placed on a cushion, fully pronated. The dorsal side of the wrist was scanned from side to side in the longitudinal plane and from superior to inferior in the transverse plane. For the wrist evaluation, the radiocarpal, radioulnar, and intercarpal joints

Abbreviations: $\mathrm{CDI}$, colour Doppler image; $\mathrm{CMCl}$, carpometacarpal joint 1; CRP, C-reactive protein; DUS, Doppler ultrasound; ESR, erythrocyte sedimentation rate; $\mathrm{HAQ}$, Health Assessment Questionnaire; $M C P$, metacarpophalangeal joint; PIP, proximal interphalangeal joint; $\mathrm{RA}$, rheumatoid arthritis; $\mathrm{RI}$, resistive index; ROI, region of interest; VAS, visual analogue scale 
were scanned. The finger joints were scanned in the longitudinal plane only and the palmar aspects were not investigated. The CMCl, MCPl, and PIP joints were scanned in an arch of $180^{\circ}$ from the ulnar to the radial side. MCP2-5 were scanned in the regions that were accessible from the dorsal side: MCP2 and MCP5 in an arch of $150^{\circ}$ and MCP $3-4$ in an arch of $120^{\circ}$.

In this study, synovial hypertrophy or the synovial volume was not estimated as previously described, ${ }^{9}$ as the aim was to obtain the Doppler images only. No normal values so far exist for the thickness of the normal synovial membrane, and it is difficult to differentiate between cartilage and synovial membrane both in patients with arthritis and in normal joints. The cartilage is therefore included in the joint volume and we see this as a systematic error. However, as there is no vascularisation of the cartilage it does not have any influence on the Doppler findings.

\section{Colour Doppler}

Blood flow in the synovial membrane was visualised with colour Doppler, which is as sensitive as the power Doppler on the Sequoia ${ }^{\circledR}$. The colour Doppler settings were the same for all joints and all volunteers, with a gain setting just below the noise level using our settings for low flow (Nyquist limit $\pm 0.014 \mathrm{~m} / \mathrm{s}$ and $7 \mathrm{MHz}$ Doppler frequency). With these settings, all the colour pixels in the image correspond to motion-that is, blood flow. That the colour pixels represented flow and not an artefact was established with the spectral Doppler displaying arterial or venous flow or, in the few cases where the signal was too weak for spectral analysis, by the constant position of the colour pixels inside the synovial membrane. This in contrast to noise or movement artefacts that appear in random fashion and in clear contrast to artefacts generated at highly reflective surfaces.

Quantitative estimation of the vascularisation in the synovial membrane was performed using the selected colour Doppler image (CDI) with maximum colour activity. The digitally stored CDI in DICOM format was transferred to a processing program (a beta version of DataPro ${ }^{\mathrm{TM}}$; Noesis, Courtaboeuf, France). The region of interest (ROI) was traced on the synovial membrane. On the US image the synovial membrane appears predominantly hypoechoic, covering the bone surfaces adjacent to the joint and superficially bounded from the subcutaneous tissue by a thin white strand, the fibrous capsule. The US joint anatomy is illustrated in fig 1. The number of colour pixels and the total number of pixels in the ROI were counted, ${ }^{5}$ and the number of colour pixels was then expressed in relation to the total number of pixels in the ROI as the colour fraction.

\section{SPECTRAL DOPPLER}

Previous experience with both the pulsatility and resistive indices ${ }^{5}$ have lead us to work only with the resistive index (RI) as it is easy to understand.

Using colour Doppler as guidance, the spectral Doppler sampling area was placed over an intra-synovial artery and the spectrum was obtained. An intra-muscular artery in the thenar muscles was sampled as a reference. The US unit traced the Doppler spectrum electronically, and identified the cardiac cycles, peak systolic flow, and end diastolic flow. The unit then calculated the RI as (peak systolic flow minus end diastolic flow)/peak systolic flow. If more than one synovial artery could be sampled, a mean RI was used. With the spectral Doppler technique it is possible to evaluate the type of flow-that is, low peripheral resistance $v$ high peripheral resistance, in the synovial membrane. The degree of peripheral resistance is expressed numerically with the RI. ${ }^{12}$ The RI has been used in obstetrics and in nephrology, to give a numerical value for the diastolic component in the umbilical arteries and arteries of the transplanted kidney. ${ }^{13}{ }^{14}$ In rheumatology, low values of RI mean low resistance, indicating inflammation, whereas high values mean high resistance, which is normal in resting musculoskeletal tissues. ${ }^{5}$ Because the intra-synovial vessels are very small, both the artery and its concomitant veins are often sampled simultaneously even with the smallest possible Doppler gate. A flow reversal during diastole (normal in musculoskeletal tissues) will then go unnoticed because the reversed arterial flow will drown in the venous signal. In order to obtain uniform measurements, we therefore limited the spectral measurements to the arterial side of the Doppler line and defined 1.00 as the maximum for RI.

When spectral Doppler measurements could not be measured, the RI was noted to be the same as in the thenar muscles, as the resistance in the synovial arteries was presumed to be the same as extra-synovial musculoskeletal flow. This assumption has been made for statistical reasons to obtain a value for normal RI in the cases where no flow was present on colour Doppler. No flow on colour Doppler is a normal finding and for RI we therefore used a value obtained from normal resting musculoskeletal tissue-that is, the thenar muscles in this study.

Erosions visualised by US were defined as a disruption of the bone surface by an indentation, which gave the bone surface an irregular appearance. ${ }^{15}$ Osteophytes were defined as bone extensions from non-cartilaginous bone surfaces inside the joint space.

\section{STATISTICS}

Statistical analysis was performed using the SPSS program (version 10.0). For correlation between age and US parameters and between the two US parameters, Pearson's test was used. Student's $t$ test was used for evaluation of the total dataset. Level of significance was $\mathrm{p}=0.05$.

\section{RESULTS}

There were no differences between the US findings in healthy men and women. The DUS findings for the individual joints are shown in table 1. The mean RI and the colour fraction showed a statistically significant correlation, with $\mathrm{R}$ values of $-0.518(\mathrm{p}<0.01)$.

Mean (SD) (range) RI for all joints for the healthy volunteers was $1.00(0.06)(0.47-1.00)$ and the colour fraction 0.008 (0.03) (0-0.32). The mean (range) RI from the thenar muscles was $1.00(1.00-1.00)$.

Colour Doppler activity was detected in $11 \%(37 / 324)$ of the joints (fig 2). Of these, it was possible to obtain spectral Doppler signals in 21 joints. Mostly, only one solitary artery could be sampled and in only three joints (two wrists and one CMCl joint) could three arteries be sampled.

There was a weak, but statistically significant correlation between age and colour fraction ( $R$ value $0.163 ; p<0.01$ ), but this correlation was not significant if the oldest subject was excluded. We found no correlation between age and RI.

Small erosions were found in three CMCl joints but not in any other joints and in no particular age group. In these three joints there was colour Doppler activity in one (with the highest colour fraction (0.32)) and three arteries detected with spectral Doppler (mean RI 0.57). OA changes (osteophytes) were found in two CMC1 joints and in 12 PIP joints in seven subjects, all $>52$ years of age. The two CMCl joints had colour Doppler activity with colour fractions of 0.01 and 0.02. Both had measurable flow with RI of 1.00 and 0.71 . None of these OA joints had erosions.

\section{DISCUSSION}

The present study demonstrated a presence of Doppler activity in $11 \%$ of the hand and finger joints of healthy 


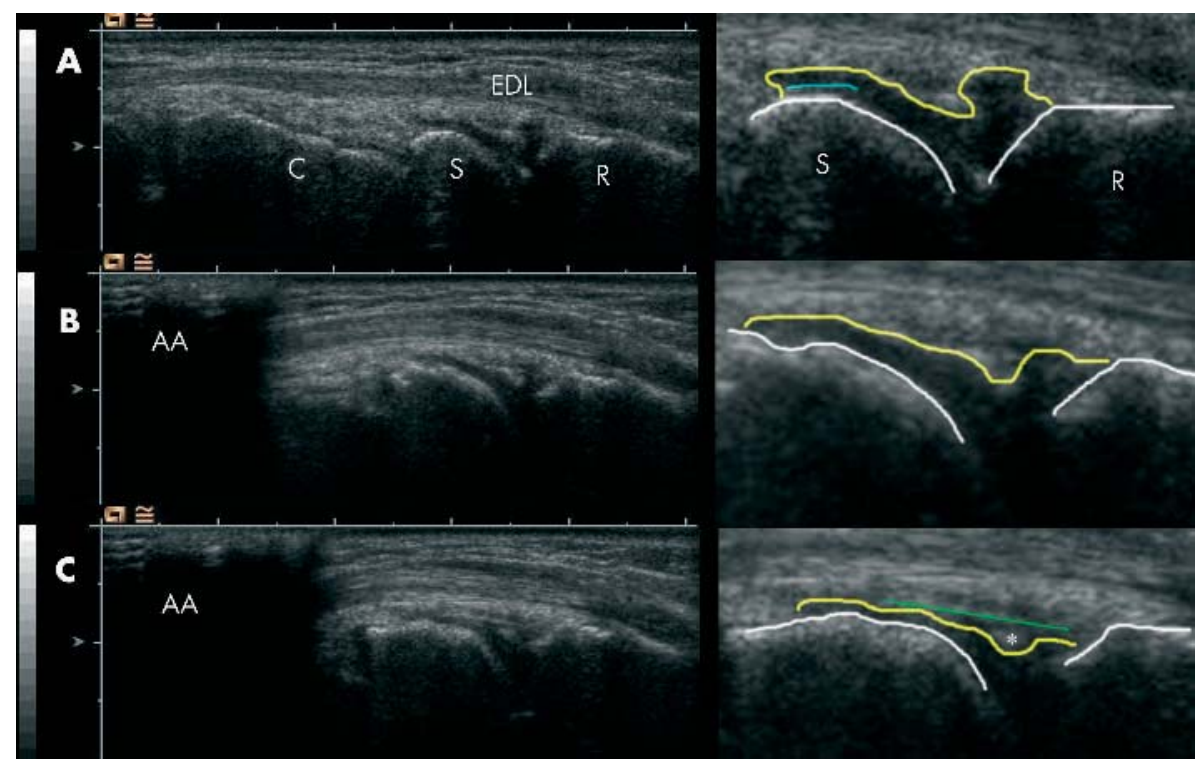

Figure 1 The synovial membrane of the radiocarpal joint during flexion. Three images have been selected from a live clip of $38 \mathrm{images.} \mathrm{Images} \mathrm{A}$ to C span from neutral position (A), over medium flexed (B), to fully flexed (C). R, radius; S, os scaphoideum; C, os capitatum; EDL, extensor digitorum longus tendon. Because the dorsal surface of the wrist becomes convex during flexion, the flat linear array transducer is unable to maintain contact over the whole sole of the transducer and an air artefact (AA) is seen in the distal third of the image in band C. Three corresponding magnified views of the radioscaphoid interaction are seen to the right. The exterior border of the synovial membrane is traced with a yellow line. In the top image, the visible part of the cartilage on the scaphoid is indicated with the blue line. As usual, the cartilage is only visible when insonated at angles close to $90^{\circ}$. More centrally on the curved surface of the scaphoid, the cartilage is indistinguishable from the synovial membrane. In neutral, the synovial membrane is folded with a proximal and distal duplication. During flexion, the synovial membrane is thinned as the duplications are mobilised. In the fully flexed position, the fibrous capsule becomes visible as a thin straight white line indicated with a green line in the magnified view. A small reflective triangle (*) is visible in all images and in the bottom image it is apparent that it lies deep in the fibrous capsule. It is therefore part of the synovial membrane and is probably a fat pad. The images demonstrate two systematic errors: we include the cartilage in the synovial trace and we do not include fat pads mentioned above (because they were only identifiable in situations where the fibrous capsule is visible).

volunteers with no history of arthritis, hand or wrist trauma, or current symptoms. Although the majority of the joints examined were without Doppler activity, we believe the $11 \%$ positive in this study to be substantial, disqualifying the mere presence of colour pixels on scans as an indication of inflammation.

Anatomically, the synovial membrane consists of a lining layer and a sub-lining layer bordering on the fibrous capsule. Deep in the sub-lining, arteries and veins can be seen, which decrease in size as the lining layer is approached. ${ }^{16}$ Detection of arterial flow in the synovial membrane depends on the sensitivity of the Doppler equipment, and our findings of colour pixels in normal synovial membrane scans are in accordance with one previous study reporting colour pixels in joint scans of 15 medical students, ${ }^{17}$ but in contrast to other studies reporting negative Doppler findings in healthy controls. ${ }^{18}{ }^{19}$

Table 1 Doppler findings in healthy volunteers

\begin{tabular}{lllll}
\hline & $\begin{array}{l}\text { No of joints } \\
\text { with colour } \\
\text { fraction/ } \\
\text { total no. } \\
\text { investigated (range) }\end{array}$ & $\begin{array}{l}\text { Colour fraction } \\
\text { Jolour } \\
\text { colour } \\
\text { Doppler+ } \\
\text { joints with } \\
\text { measurable } \\
\text { RI }\end{array}$ & Mean RI (range) \\
\hline Wrist & $15 / 27$ & $0.08(0.01-0.23)$ & $8 / 15$ & $0.85(0.47-1.00)$ \\
CMC1 & $11 / 27$ & $0.08(0.01-0.32)$ & $9 / 11$ & $0.85(0.51-1.00)$ \\
MCP & $10 / 135$ & $0.05(0.01-0.11)$ & $3 / 10$ & $0.85(0.77-1.00)$ \\
PIP & $1 / 135$ & 0.12 & $1 / 1$ & 0.67 \\
Total & $37 / 324$ & & $21 / 37$ & \\
\hline
\end{tabular}

The difference between the colour Doppler findings in the $\mathrm{CMCl}$ and the MCP joints were statistically significant at $p<0.001$.

Doppler+, Doppler positive.
Doppler US is a relatively new tool in the investigation of local inflammation in arthritis. ${ }^{20}$ The Doppler signals are a reflection of the number of erythrocytes that move within the image plane and the speed with which they move. In consequence, the colour pixels are an estimate of the amount of moving blood in the investigated area. ${ }^{21} 22$ Previous studies have shown that there is a correlation between the histological and sonographical degree of vascularity; ${ }^{4}$ however the number of colour pixels do not correspond to the number of vessels visualised by DUS but rather to the perfusion of these vessels. ${ }^{3}$ A correlation between US and MRI has also been determined recently, ${ }^{18}$ and the inflammatory parameters used in this study has been validated in a recent study comparing US and MRI, in which both symptomatic and asymptomatic joints were examined. ${ }^{23}$

The presence of colour pixels on scans of the synovial membrane has until now been taken as a sign of hyperaemia, ${ }^{8}$ but with increasing Doppler sensitivity, normal synovial flow may also result in colour pixels. Accordingly, the mere presence of colour pixels cannot at present be interpreted as a sign of synovitis per se, especially in the wrists and to some extent in the MCP joints as indicated by our study. The number of colour pixels seen in our scans is comparable to that which we frequently encounter sporadically when we scan the asymptomatic joints of the hands of an RA subject. We do not consider it a sign of pathology, and as we show here, these findings can also be seen in healthy volunteers. Spectral Doppler examinations on joints of healthy volunteers have, to our knowledge, not been previously investigated. The spectral Doppler examination depends on colour Doppler to identify the presence and location of vessels and was therefore only applied in the 37 colour Doppler positive joints in this study. In 21 of these 


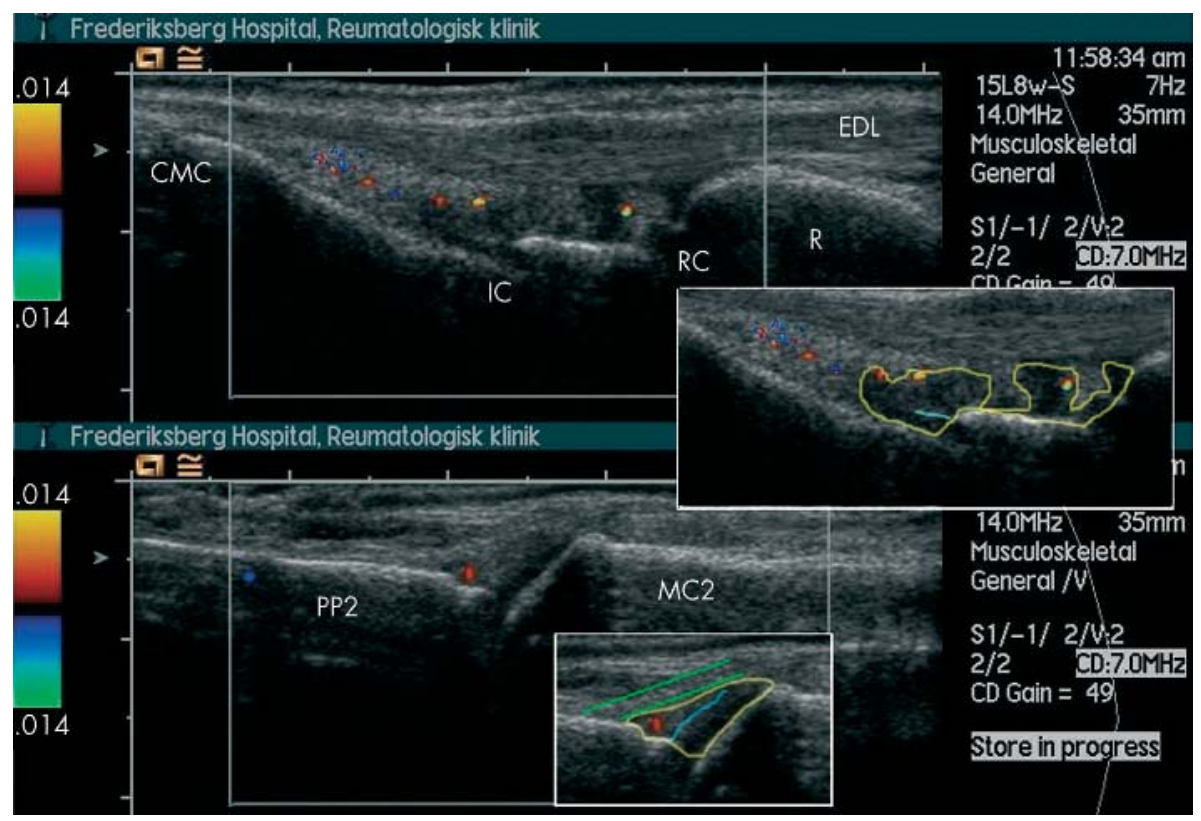

Figure 2 Colour Doppler activity in wrist and MCP. The top image is a longitudinal scan in the middle of the wrist with the extensor digitorum longus tendon (EDL). The distal part of the radius (R) is seen in the right side of the image. On the inset, the synovial membrane is traced with a yellow line and is seen as hypo-echoic (dark) protusions from the radiocarpal joint (RC) and the intercarpal joint (IC). There is a single colour spot in the proximal protusion and two in the distal. The surface of the cartilage on the os capitatum is traced with blue. The carpometacarpal joint (CMC) is seen in left side of the image. The bottom image is a longitudinal scan of an MCP2 joint. The metacarpal bone (MC2) is in the right side of the image and the proximal phalanx (PP) in the left. There is a single colour spot in the synovial membrane just distal to the joint space. The inset shows the membrane traced with yellow following the bone surfaces and the underside of the fibrous capsule (two green lines). The cartilage on the metacarpal head (traced with blue) is included in the ROI in order to obtain uniform measurements. In inflamed joints where the synovial membrane is hypo- or an-echoic, we usually could not identify the cartilage.

joints, it was possible to identify an artery and thereby obtain an RI.

In normal resting musculoskeletal tissues, flow is seen with colour or power Doppler in muscles and connective tissue, seldom in joints, and never in tendons. When the flow is assessed by spectral Doppler, no diastolic flow is found in these tissues at rest and consequently, the RI is 1.00. This was demonstrated in this study by all 27 subjects having an RI of 1.00 in the thenar muscles. In rheumatology, low values of RI indicate pathology, which has been observed in previous studies. ${ }^{5} 820$

Among the healthy volunteers only one synovial artery could be sampled from the investigated joints, apart from two wrists and one $\mathrm{CMCl}$ joint in which three synovial arteries could be sampled. In general, when examining RA patients with our method, inflamed joints have three or more different synovial arteries that can be sampled. ${ }^{5}{ }^{10}$ The present findings show that even though low RI values may be obtained from healthy volunteers, our mean values $(0.85)$ are higher than those reported in RA patients ${ }^{24} 0.4-0.7$ and $0.65-0.76 .^{20}$

The positive Doppler findings in some normal joints indicate that the mere presence of colour pixels on scans may not be enough for diagnosis of inflammation. In chronic synovitis we also find greyscale abnormalities such as synovial proliferation and/or effusion, but if DUS is to be used in the detection of inflammatory changes in very early arthritis it is not likely in those situations to find synovial proliferation and/or effusion. Therefore, it seems rational that a cutoff level (above which inflammation is present) for both colour fraction and RI should be established for diagnostic purposes rather than depending on the more or less static greyscale changes.

In these asymptomatic subjects, we found few greyscale changes indicating OA. These changes were predominantly in
PIP joints and not accompanied by colour Doppler activity. In the two CMCl joints with osteophytes we did find colour Doppler activity (colour fraction of 0.01 and 0.02 ) and in one joint a low RI (0.71) also. All these joints were identified in subjects over 50 years of age. The only signs of erosive changes in healthy volunteers were found in the CMCl joint (three cases), which is also prone to OA changes. In the joints recommended by the ACR to be evaluated in $R^{25}$ we found no erosive changes, indicating that the presence of erosions by US in the wrist, MCP, and PIP joints are signs of pathology.

Our findings show that colour Doppler activity is most common in the wrist (15/27) followed by CMCl (11/27), MCP (10/135), and very seldom in the PIP joints (1/135). The rather frequent observation of positive findings in the wrist may be due to the composite structure of this joint. If it is assumed that joints of comparable size have the same chance of displaying colour Doppler activity, the wrist is naturally more likely because it is a group of joints. In this respect, $\mathrm{CMCl}$ has remarkably high colour Doppler activity, which, according to joint size, should be closer to the MCP joints. In this study, the CMCl may therefore be seen as an outlier not only in terms of colour Doppler activity but also in OA changes and erosions. In accordance with the ACR recommendations from the clinical point of view, the ultrasonographic changes are in support of an exclusion of this joint from a diagnostic evaluation of arthritis by imaging.

\section{CONCLUSION}

Colour Doppler activity may be found in healthy volunteers, most commonly in the wrist, more rarely in the MCP joints, and hardly ever in the PIP joints. The CMCl joint is prone to both greyscale and Doppler findings not necessarily related to $\mathrm{RA}$ and therefore it is reasonable that this joint should not be 
part of the recommended joint evaluation. The Doppler sensitivity is increased in newer US machines and seems to approach the detection limit of normal synovial flow. As sensitivity is likely to continue with development of new machines, it will be necessary in the near future to be able to distinguish normal from pathological synovial flow.

\section{Authors' affiliations}

L Terslev, S Torp-Pedersen, E Qvistgaard, P von der Recke, H Bliddal, The Parker Institute, Department of Rheumatology, Frederiksberg Hospital, DK 2000 Frederiksberg, Denmark

\section{REFERENCES}

1 Backhaus M, Kamradt T, Sandrock D, Loreck D, Fritz J, Wolf KJ, et al. Arthritis of the finger joints: a comprehensive approach comparing conventional radiography, scintigraphy, ultrasound, and contrast-enhanced magnetic resonance imaging. Arthritis Rheum 1999;42:1232-45.

2 Newman JS, Adler RS, Bude RO, Rubin JM. Detection of soft-tissue hyperemia: value of power Doppler sonography. AJR Am J Roentgenol 1994; 163:385-9

3 Schmidt WA, Volker L, Zacher J, Schlafke M, Ruhnke M, Gromnica-Ihle E. Colour Doppler ultrasonography to detect pannus in knee joint synovitis. Clin Exp Rheumatol 2000;18:439-44.

4 Walther M, Harms H, Krenn V, Radke S, Faehndrich TP, Gohlke F. Correlation of power Doppler sonography with vascularity of the synovial tissue of the knee joint in patients with osteoarthritis and rheumatoid arthritis. Arthritis Rheum 2001:44:331-8.

5 Qvistgaard E, Rogind H, Torp-Pedersen S, Terslev L, Danneskiold-Samsoe B Bliddal H. Quantitative ultrasonography in rheumatoid arthritis: evaluation of inflammation by Doppler technique. Ann Rheum Dis 2001;60:690-3.

6 Stone M, Bergin D, Whelan B, Maher M, Murray J, McCarthy C. Power Doppler ultrasound assessment of rheumatoid hand synovitis. J Rheumatol 2001;28:1979-82.

7 Newman JS, Laing TJ, McCarthy CJ, Adler RS. Power Doppler sonography of synovitis: assessment of therapeutic response-preliminary observations. Radiology 1996; 198:582-4

8 Terslev L, Torp-Pedersen S, Qvistgaard E, Danneskiold-Samsoe B, Bliddal H. Estimation of inflammation by Doppler ultrasound: Quantitative changes after intra-articular treatment in rheumatoid arthritis. Ann Rheum Dis 2003;62:1049-53.

9 Hau M, Kneitz C, Tony HP, Keberle M, Jahns R, Jenett M. High resolution ultrasound detects a decrease in pannus vascularisation of small finger joints in patients with rheumatoid arthritis receiving treatment with soluble tumour necrosis factor alpha receptor (etanercept). Ann Rheum Dis 2002;61:55-8.

10 Terslev L, Torp-Pedersen S, Qvistgaard E, Kristoffersen H, Røgind H, Danneskiold-Samsøe B, et al. Effects of treatment with etanercept (ENBREL,
TNRF:Fc ) on rheumatoid arthritis evaluated by Doppler ultrasonography. Ann Rheum Dis 2003;62:178-82

11 Torp-Pedersen T, Torp-Pedersen S, Bliddal H. Diagnostic value of ultrasonography in epiconylitis. Ann Intern Med 2002;136:781-2.

12 Pourcelot L. L'examen Doppler des vaisseaux périphériques. Paris: AC-D Production, 1982

13 Skjoldbye B, Nielsen AH, Court-Payen, Norgaard N, Rasmussen F, Lokkegaard $\mathrm{H}$, et al. Perioperative Doppler ultrasonography: renal detection of renal graft perfusion. Scand J Urol Nephrol 1998;32:345-9.

14 Struijk PC, Ursem NT, Mathews J, Clark EB, Keller BB, Wladimiroff JW. Power spectrum analysis of heart rate and blood flow velocity variability measured in the umbilical and uterine arteries in early pregnancy: a comparative study. Ultrasound Obstet Gynecol 2001;17:316-21.

15 Backhaus M, Burmester GR, Sandrock D, Loreck D, Hess D, Scholz A, et al. Prospective two year follow up study comparing novel and conventional imaging procedures in patients with arthritic finger joints. Ann Rheum Dis 2002;61:895-904.

16 Davies D, Edwards D. The blood supply of the synovial membrane and the intra-articular structures. Ann Royal Coll Surg Engl 1948:142-56.

17 Hau M, Schultz H, Tony HP, Keberle M, Jahns R, Haerten R, et al. Evaluation of pannus and vascularization of the metacarpophalangeal and proximal interphalangeal joints in rheumatoid arthritis by high-resolution ultrasound (multidimensional linear array). Arthritis Rheum 1999;42:2303-8.

18 Szkudlarek M, Court-Payen, Strandberg C, Klarlund M, Klausen T, Ostergaard M. Power Doppler ultrasonography for assessment of synovitis in the metacarpophalangeal joints of patients with rheumatoid arthritis: a comparison with dynamic magnetic resonance imaging. Arthritis Rheum 2001;44:2018-23.

19 Klauser A, Frauscher F, Schirmer M, Halpern E, Pallwein L, Herold M, et al. The value of contrast-enhanced color Doppler ultrasound in the detection of vascularization of finger joints in patients with rheumatoid arthritis. Arthritis Rheum 2002;46:647-53.

20 Silvestri E, Martinoli C, Onetto F, Neumaier CE, Cimmino MA, Derchi LE. (Evaluation of rheumatoid arthritis of the knee with Doppler color.) Radiol Med (Torino) 1994;88:364-7

21 Rubin JM, Adler RS, Fowlkes JB, Spratt S, Pallister JE, Chen JF, et al. Fractional moving blood volume: estimation with power Doppler US. Radiology 1995; 197:183-90.

22 Rubin JM, Bude RO, Carson PL, Bree RL, Adler RS. Power Doppler US: a potentially useful alternative to mean frequency-based color Doppler US Radiology 1994; 190:853-6.

23 Terslev L, Torp-Pedersen S, Savnik A, Von der Recke P, DanneskioldSamsoe B, Bliddal H. Doppler ultrasound and MRI of synovial inflammation in the hand in rheumatoid arthritis patients - a comparative study. Arthritis Rheum 2003:48:2434-41.

24 Midiri M, lovane A, Finazzo M, Brancatelli G, Gallo C, Lagalla R. (Color Doppler-echo in rheumatoid arthritis with extra-articular location. Preliminary experience.) Radiol Med (Torino) 1999;98:123-6.

25 Arnett FC, Edworthy SM, Bloch DA, McShane DJ, Fries JF, Cooper NS, et al. The American Rheumatism Association 1987 revised criteria for the classification of rheumatoid arthritis. Arthritis Rheum 1988;31:315-24. 